\title{
A comparison of the changes in suprathreshold apparent contrast in acute and simulated optic neuritis
}

\author{
M ABRAHAMSSON, J SJÖSTRAND \\ From the Department of Ophthalmology, University of Göteborg, Sweden.
}

SUMMARY A thin PVC film was used as a diffusion filter together with glass lenses in order to induce a unilateral artificial contrast sensitivity depression in controls. These artificially depressed contrast sensitivity functions were made comparable to those assessed in patients during the recovery phase of their acute unilateral optic neuritis. A technique of subjective suprathreshold contrast matching was used to determine the suprathreshold apparent contrast in patients and controls with acute and simulated optic neuritis respectively. The results showed that differences in apparent contrast between the eyes is proportional to the discrepancy in threshold contrast for high and intermediate spatial frequencies in patients with optic neuritis. For low frequencies the apparent contrast difference was independent of threshold differences. Contrast vision of controls with simulated optic neuritis did not show this frequency dependency. There was also a discrepancy in visual acuity between the two groups. Generally the control with artificially depressed contrast sensitivity functions has a letter acuity value lower than that of the optic neuritis patient with a corresponding CSF depression.

Letter acuity is the most common method used to study the capability of the visual system to detect and process spatial information. It represents recognition of a suprathreshold target at a high spatial frequency. More information about the detection ability is obtained by the contrast sensitivity function, which represents the threshold contrast for different spatial frequencies. Knowledge of the ability of the visual system to process contrasts above the threshold at different spatial frequencies is necessary in order to understand how every day images are perceived. Neither contrast sensitivity function nor acuity give direct access to how suprathreshold spatial information in general is perceived. The fact that threshold and suprathreshold contrast processing can differ has been shown for normal vision ${ }^{12}$ as well as for patients with amblyopia. ${ }^{3}$ Our knowledge about the suprathreshold vision in different diseases is limited. In order to gain more insight into the visual perception of the impaired visual system we need to investigate the suprathreshold contrast vis-

Address for reprint requests: $M$ Abrahamsson, Department of Ophthalmology, Sahlgren's Hospital, S-413 45 Göteborg, Sweden.

Received 4 January 1985 and in revised form 13 March 1985. Accepted 23 March 1985 ion and not just the threshold contrast. In an earlier work $^{4}$ we studied suprathreshold vision in acute optic neuritis and found that the disturbance of the suprathreshold contrast perception was dependent on spatial frequency. Hess studied in a similar way patients with remaining deficits, when recovered from optic neuritis and did not find any frequencydependent suprathreshold vision disturbance. ${ }^{5}$ This discrepancy is probably because the patients in our study were in the acute phase of optic neuritis.

In this study we have compared suprathreshold vision in patients with optic neuritis with that of controls with contrast sensitivity function artificially depressed and matched to the deficits of the various optic neuritis patients. The aim of the study was to determine whether the frequency-dependent suprathreshold contrast vision found in acute optic neuritis was related to the CSF depression or if the suprathreshold contrast vision was independent of the contrast sensitivity function change.

\section{Method}

Patients. Seven controls (age $20-40 \mathrm{yr}$ ) and seven patients with unilateral retrobulbar optic neuritis (aged 25-35 yr) took part in this study. Matching data from four of the patients in a previous study ${ }^{4}$ have been replotted in log-log 
coordinates and included in the analysis. Each patient was submitted to a careful ophthalmological examination. Monocular visual acuity was determined with optimal correction at a test distance of $5 \mathrm{~m}$ using a natural pupil. An officially approved acuity chart (KIFA: MonoyerGranström) illuminated with approximately $700 \mathrm{~cd} / \mathrm{m}^{2}$ in a LIC universal light box was used. All controls had a corrected vision that was $1.0(20 / 20)$ or better. A $100 \%$ criterion was used.

Contrast sensitivity. Vertical, stationary and sinusoidal gratings were generated on a TV monitor as previously described. ${ }^{67}$ The threshold contrast was determined monocularly by raising the contrast from a subthreshold setting as a selected spatial frequency until a grating is faintly seen. This was done for both eyes using optimal optical correction. The television screen was masked to subtend $6^{\circ} \times 6^{\circ}$ of angle at the eye, when viewed at a distance of $2.5 \mathrm{~m}$. Sixteen frequencies between $0 \cdot 5-39$ cycles per degree were explored twice at random. The contrast was raised in steps of $2 \mathrm{~dB}$ within the contrast range 0.001 to 0.7 .

Simulation. The contrast sensitivity function was depressed unilaterally in controls in order to resemble that of patients with retrobulbar optic neuritis obtained at different stages of their recovery. This was done by using a combination of lenses and filters. The filter material, referred to commercially as unplasticised (flexible) PVC white, was available in sheet of thickness $0.127 \mathrm{~mm} .^{8}$ One of the controls had his contrast sensitivity function measured for several combinations of lenses and filters. These results could then be used to get a fairly good approximation of the lens/filter combination needed for a certain contrast sensitivity function depression. Only small individual corrections were needed in order to match a contrast sensitivity function from a certain optic neuritis patient at all spatial frequencies $(0.5$ to 39.0 cycles per degree) with the artificially depressed contrast sensitivity function from a control.

Suprathreshold contrast. The apparent suprathreshold contrast was measured by presenting one of two vertical, sinusoidal gratings with identical spatial frequency, to each eye. The equipment has previously been described. ${ }^{4}$ To test the validity of this method we also used a suprathreshold grating presented on a TV monitor as a reference grating in some experiments and compared the results to those obtained with a photographical reference grating. There was no difference between the results obtained with the two methods. The head of the patient was fixed by means of a chin support $2.5 \mathrm{~m}$ in from of the gratings. The non-variable reference grating was presented for the eye which had an artificially depressed contrast sensitivity function or the eye with the optic neuritis. The grating with the variable contrast was presented to the contralateral eye. The patients wore goggles with polarising filters. In front of the test targets were also polarising filters. The polarising angle was set in order to make only the selected grating visible to the right or left eye. The adaptation effect was minimized by placing the gratings $20^{\circ}$ apart. The patient was then forced to look at one target at the time and it was necessary to make rapid eye movements during the contrast matching. The patient's task was to adjust the contrast of the variable grating with a continuous attenuator until it matched the non-variable reference grating in apparent contrast. Three different spatial frequen- cies were examined $(0.7,2.7$ and 6.7 cycles per degree). The reference grating had three contrast levels $(0 \cdot 15,0 \cdot 30$ and $0 \cdot 80$ ). The patients matched each contrast five times at each spatial frequency. The controls did one test session where contrast matching was done with optimal correction on both eyes and two test sessions at which the right eye was artificially depressed. During the test session where optimal correction was used, first all the reference gratings were presented to the left eye and then they were all presented to the right eye. Retesting within the same day demonstrated a range of contrast matching that was less than $\pm 10 \%$. Each setting of a contrast match rarely took more than 10 seconds and a grating was in general inspected less than two seconds at a time before the eye changed position. The controls with artificially depressed contrast sensitivity function in general needed slightly more time to do each setting.

\section{Results}

\section{Evaluation of the method}

It is evident that controls, optimally corrected, can make an accurate and rapid contrast matching of two gratings of identical spatial frequency presented one to each eye. In the frequency range 0.7 to 6.2 cycles per degree the matching is both proportional and accurate (fig 1).

Contrast sensitivity function. Each optic neuritis patient had his contrast sensitivity function measured at three or four test sessions during recovery (fig 2a). The contrast sensitivity function showed a depression without peak-shift that is typical for optic neuropathies. ${ }^{6}$ In the controls we induced several artificially depressed contrast sensitivity functions in order to match them with those of the patients. Contrast sensitivity function pairs consisting of one optic neuritis patient and one control with corresponding contrast sensitivity functions were selected (fig 2). The difference between the contrast

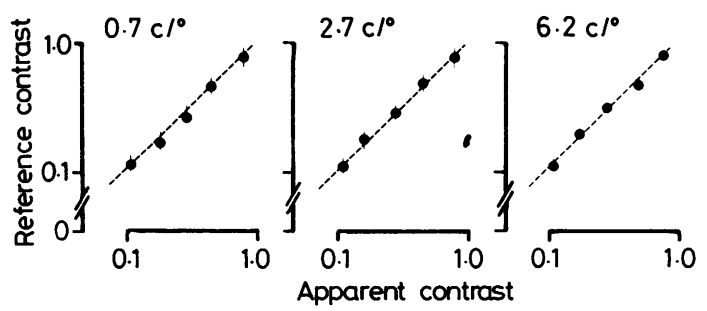

Fig 1 Suprathreshold vision was studied in controls $(n=7)$. The figure shows the results from contrast matching, plotted for three different spatial frequencies $(0 \cdot 7$, 2.7 and 6.2 cycles per degree). The circles represent the average value of seven controls. Five settings were used for each eye of each control and the reference grating was first presented to the right eye and then to the left eye. The vertical line through the circles represent $\pm 1 S D$. 


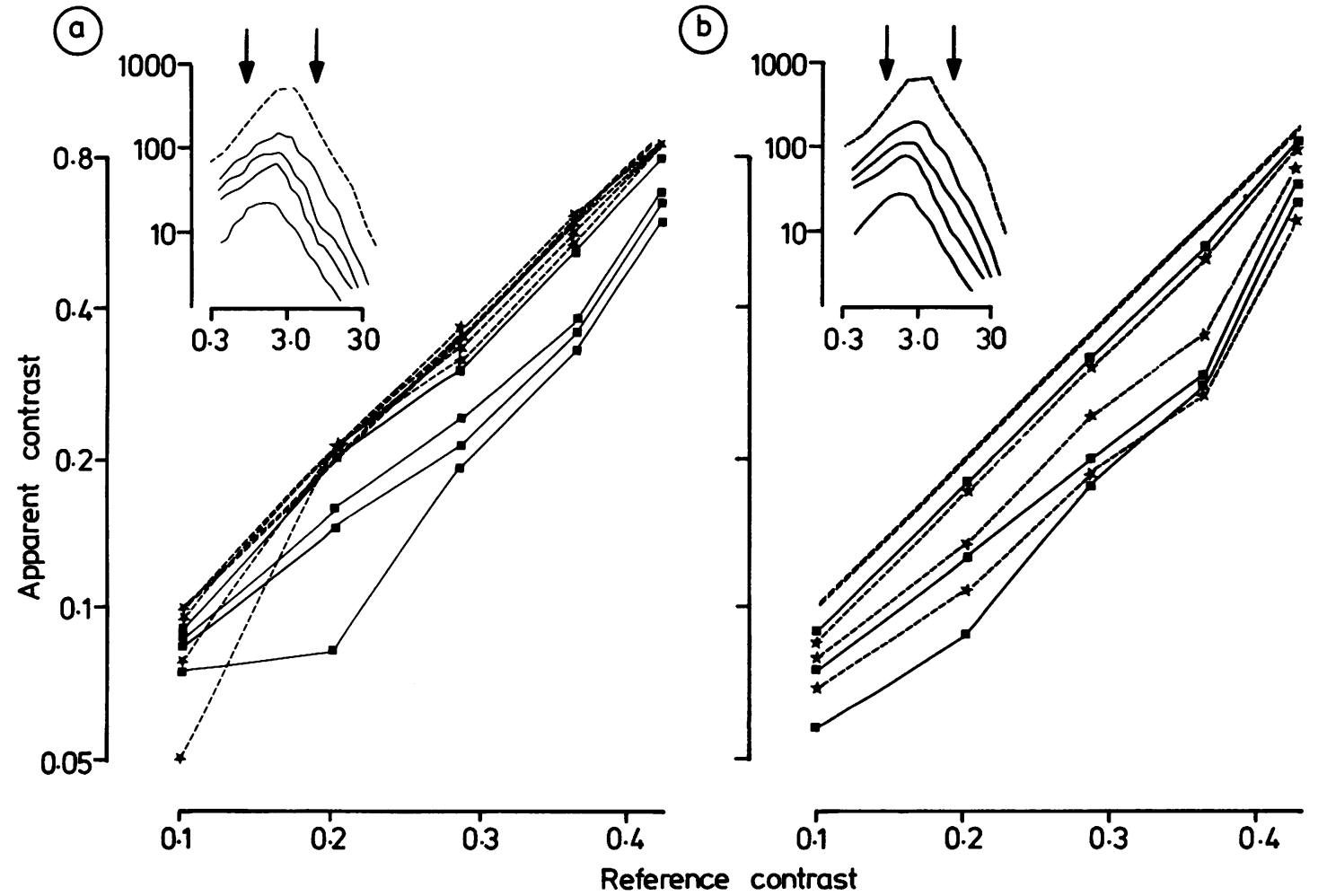

Fig 2 Suprathreshold matching data: (a) from a representative optic neuritis patient, case EH, at two spatial frequencies, 0.7 cycles per degree ( $\star$ and dashed lines); and at the frequency 6.2 cycles per degree ( $\square$ and solid lines); (b) shows the data from the simulated optic neuritis control that is matched to the patient. Apparent contrast $=$ Reference contrast is indicated by ( - Inset: contrast sensitivity functions are presented in the upper left corner. The dashed line indicates the contralateral eye and solid lines indicates the contrast sensitivity functions measured at four sessions during the recovery from acute optic neuritis (a) and for the simulated optic neuritis (b). The numbers 1-4 indicate contrast sensitivity function and suprathreshold matching data at four sessions during recovery of vision from 0.1 to 1.0 and the corresponding control. Visual acuity data are given in the table.

sensitivity functions in one such control/patient pair was not greater than $3 \mathrm{~dB}$ at any of the spatial frequencies examined.

\section{Suprathreshold contrast}

Optic neuritis patients. In all cases the suprathreshold contrast matching data were abnormal during the acute phase of the disease (fig $2 a$ ). The data are plotted in log-log coordinates. During recovery there was a normalisation of suprathreshold vision. The suprathreshold apparent contrast during the acute phase of optic neuritis was almost normal for the spatial frequency 0.7 cycles per degree while the apparent contrast at higher frequencies were severely disturbed.

The contrast matching data for two frequencies, 0.7 and 6.2 cycles per degree, obtained during recovery from the acute phase of optic neuritis from a representative patient are presented in fig $2 a$. There is a clear-cut difference between the results of the contrast matching at the two selected frequencies. At high frequency the ratio between the apparent contrast and the reference contrast at different contrast values was proportional to the difference in the threshold value (contrast sensitivity function) between the eyes. There was no evidence for such a proportionality in the low frequency data. The contrast matching seemed to be fairly accurate independent of the degree of threshold discrepancy between the eyes except for the suprathreshold contrast levels closest to the threshold value at which a proportionality between differences in contrast sensitivity function and apparent suprathreshold contrast can be traced. 
Simulated optic neuritis The controls with artificial optic neuritis showed an abnormal suprathreshold matching that in some part was similar to that of the optic neuritis patients. The data from a representative control are presented in fig $2 \mathrm{~b}$. In the highfrequency domain the ratio between the apparent contrast and the reference contrast values was proportional to the difference in threshold values between the eyes and the same was true at the low frequency. At the low frequency the main difference between the apparent contrast in optic neuritis and the simulated one is clearly shown (fig 2). In fig 3 one contrast matching for each patient and each control is presented confirming that this contrast matching difference between the two groups were present in all cases. Thus there is no principal difference between the data at high and low frequency in the simulated cases whereas the optic neuritis patients show the above mentioned sparing at a low frequency.

\section{Visual acuity}

Letter acuity data from the optic neuritis patients and the controls are presented in the table. For each patient/control pair with corresponding contrast sensitivity functions it was found that the letter acuity in all cases below the $1 \cdot 0(20 / 20)$ acuity level was lower for simulated controls than for the patients with acute optic neuritis. In some cases the acuity difference was marked.

\section{Discussion}

The matching results of this study partly confirms the results presented by Hess. ${ }^{5}$ When he studied

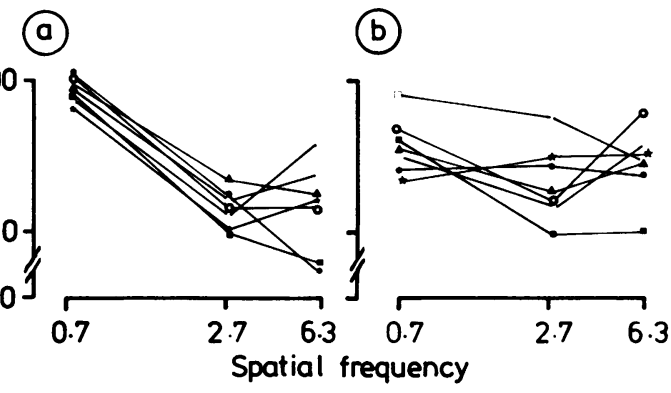

Fig 3 Suprathreshold matching data from one contrast (30\%) level at three spatial frequencies for all patients (a) and controls (b). Each optic neuritis patient and his matched simulated optic neuritis control have the same symbol.

$$
\text { Contrast ratio }=\frac{\text { apparent contrast }}{\text { reference contrast }} \times 100
$$

Table Visual acuity data for optic neuritis patients during recovery and simulated controls with corresponding contrast sensitivity function impairment

\begin{tabular}{lll}
\hline $\begin{array}{l}\text { Case } \\
\text { Optic neuritis/control }\end{array}$ & \multicolumn{1}{l}{ Visual Acuity } \\
\cline { 2 - 3 } & Optic neuritis & Control \\
\hline G. J-R. / K.L. & 0.4 & 0.1 \\
& 0.7 & 0.25 \\
& 1.0 & 1.0 \\
L-O.E. / L.M. & 0.4 & 0.3 \\
& 0.9 & 0.7 \\
E.H. / S.W. & 1.0 & 1.0 \\
& 0.1 & 0.05 \\
& 0.6 & 0.2 \\
I.B. / M.A. & 0.7 & 0.25 \\
B.W. / A-B.J. & 1.0 & 0.9 \\
T.P. / M.G. & 0.1 & 0.2 \\
I-B.B. / S.M. & 0.8 & 0.9 \\
& 1.0 & 0.7 \\
& 0.6 & 0.25 \\
& 0.9 & 0.5 \\
& 1.0 & 0.9 \\
& 0.8 & 0.7 \\
\hline
\end{tabular}

optic neuritis patients who had passed the acute phase of the disease he found that losses observed for threshold contrast stimuli are just as pronounced at suprathreshold levels. Optic neuritis is a condition in which there is a true loss of contrast sensitivity in its more general sense in cases recovered from the acute attack while it is only true for spatial frequencies greater than 0.7 cycles per degree in the acute phase. ${ }^{4}$ Our low-frequency matching results correspond to the results presented for amblyopes..$^{90}$ In a developmental anomaly such as amblyopia there is a compensatory mechanism that boosts the neural suprathreshold contrast signal. At high contrasts, well above threshold, the suprathreshold signal from the amblyopic eye is equalised to the signal from the normal eye. A similar phenomenon has been described for defocused controls at high spatial frequencies ( $>10$ cycles degree). ${ }^{\prime}$

Our study confirms that there are at least two types of suprathreshold impairment. In simulated cases there is a general impairment for all contrast levels and in acute optic neuritis there is "recruitment" at low spatial frequencies. The contrast losses we found in simulated optic neuritis can be thought of as resulting from general blurring ${ }^{11}$ of the retinal ganglion cell signal because we have defocused and diffused the retinal image whereas the blurring is non-uniform in the acute phase of optic neuritis. The results for our simulated cases resemble the data presented by Hess. ${ }^{5}$ The discrepancy between our findings in the acute phase and Hess' data from 
recovered patients with remaining deficits indicates that some changes in the relation between threshold and suprathreshold vision occurs in long-standing cases. In the present study as well as in earlier reports ${ }^{4}$ we found, unlike the findings of Hess, ${ }^{5}$ that a normalised suprathreshold vision could be combined with an abnormal threshold value. The two models for contrast coding in normal vision that were proposed by Kulikowski² (subjective contrast is directly proportional to physical contrast minus the contrast threshold) and Georgeson and Sullivan' (subjective contrast for stimuli having different contrast is equated above threshold) cannot explain our results. Hess ${ }^{5}$ postulated a relationship (subjective contrast is proportional to the logarithm of the physical contrast divided by the ratio of contrast thresholds) that described his results from recovered optic neuritis patients. It also explains the results from our simulated optic neuritis patients. These models, however, do not explain recruitment as observed in the low spatial frequency range in acute optic neuritis. ${ }^{4}$ We need another kind of model which in its simplest form may consist of two mechanisms one of which is more sensitive to contrast and/or frequency that the other. ${ }^{10}$ Alternatively there can be two mechanisms of different types, where one of them is a linear function of contrast and the other a non-linear function of contrast. ${ }^{12}$ Any of these mechanisms can be affected differently in different visual disorders and that can explain our findings of a difference in suprathreshold and threshold vision in the two groups.

The difference in visual (letter) acuity between optic neuritis patients and simulated cases with almost identical high frequency limb of the contrast sensitivity function up to 39 cycles per degree shows clearly that contrast sensitivity and visual acuity can be differently affected. The discrepancy between letter acuity deterioration between the two groups may be explained by the fact that optic neuritis patients have a generalised depression combined with localised areas of more profound sensory loss. ${ }^{13}$ This might result in islands of relatively preserved function useful for letter recognition. Another possible explanation is derived from the way we made the artificial contrast sensitivity function depression. We used diffusion filters which induce a scatter of the light and that is not presented for the optic neuritis patients.
In conclusion our study demonstrated that suprathreshold apparent contrast and visual acuity can be differently affected at corresponding contrast sensitivity functions.

This work was supported by the Carmen and Bertil Regnér foundation and by the Swedish Medical Research Council (grant 02226). We also acknowledge support from the Handlanden Svenssons Foundation.

\section{References}

${ }^{1}$ Georgeson MA, Sullivan GD. Contrast constancy: Deblurring in human vision by spatial frequency channels. J Physiol (Lond) 1975;252:627-56.

${ }^{2}$ Kulikowski JJ. Effective contrast constancy and linearity of contrast sensation. Vision Res 1976;16:1419-31.

${ }^{3}$ Hess RF, Bradley A, Piotrowski L. Contrast coding in amblyopia: I Differences in the neural basis of human amblyopia. Proc $R$ Soc Lond (Biol) 1983;217:30930.

${ }^{4}$ Sjöstrand J, Abrahamsson M. Suprathreshold vision in acute optic neuritis. J Neurol Neurosurg Psychiatry 1982;45:227-34.

${ }^{5}$ Hess RF. Contrast vision in optic neuritis: neural blurring. J Neurol Neurosurg Psychiatry 1983; 46: $1023-30$.

${ }^{6}$ Frisén L, Sjöstrand J. Contrast sensitivity in optic neuritis. A preliminary report. Docum Ophthalmol Proc Ser 1978;17:165-74.

${ }^{7}$ Sjöstrand J. Contrast sensitivity in macular disease using a small-field and a large field TV-system. Acta Ophthalmol 1979;57:832-45.

${ }^{8}$ Liubinas J. A functional analysis of methods of simulating low vision. Thesis, University of Melbourne 1979; Chapter 3.2.2.2.

${ }^{9}$ Hess RF, Bradley A. Contrast perception above threshold is only minimally impaired in human amblyopia. Nature 1980;287:463-4.

${ }^{10}$ Hess RF. Contrast-coding in amblyopia. II On the physiological basis of contrast recruitment. Proc $R$ Soc Lond (Biol) 1983;217:331-40.

"Hess RF, Wood G. Vision through cataracts. Inv Ophthalmol Vision Sci 1978;17:428-35.

12 Swanson WH, Wilson HR, Giese SC. Contrast matching data predicted from contrast increment threshold. Vision Res 1984;24:63-75.

${ }^{13}$ Frisén L. Clinical features of optic neuritis standard examination techniques. Bull Soc Belge Ophthalmol 1983;208-1:131-42. 